\title{
Exchange-spring behavior in epitaxial hard/soft magnetic bilayer films
}

\author{
J.S. Jiang, Eric E. Fullerton, ${ }^{*}$ M. Grimsditch, C.H. Sowers, and S.D. Bader
}

Materials Science Division

Argonne National Laboratory, Argonne, IL 60439

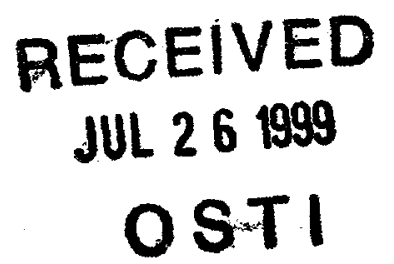

43rd Annual Conference on Magnetism and Magnetic Materials, January 6-9, 1998, San Francisco, CA

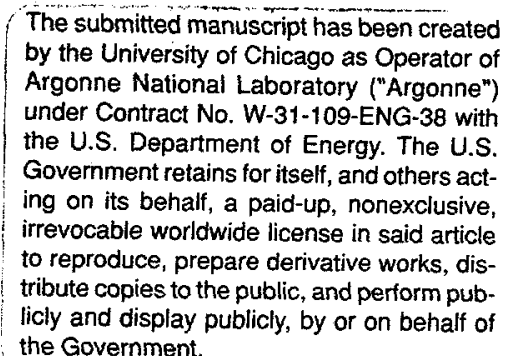

Work supported by the U.S. Department of Energy, Basic Energy Sciences-Materials Sciences under contract \#W-31-109-ENG-38.

*Present address: IBM Almaden Research Center, 650 Harry Rd., San Jose, CA 95120-6099. 


\section{DISCLAIMER}

This report was prepared as an account of work sponsored by an agency of the United States Government. Neither the United States Government nor any agency thereof, nor any of their employees, make any warranty, express or implied, or assumes any legal liability or responsibility for the accuracy, completeness, or usefulness of any information, apparatus, product, or process disclosed, or represents that its use would not infringe privately owned rights. Reference herein to any specific commercial product, process, or service by trade name, trademark, manufacturer, or otherwise does not necessarily constitute or imply its endorsement, recommendation, or favoring by the United States Government or any agency thereof. The views and opinions of authors expressed herein do not necessarily state or reflect those of the United States Government or any agency thereof. 


\section{DISCLAIMER}

Portions of this document may be illegible in electronic image products. Images are produced from the best available original document. 


\title{
Exchange-spring behavior in epitaxial hard/soft magnetic bilayer films
}

\author{
J. S. Jiang, Eric E. Fullerton*, M. Grimsditch, C. H. Sowers, and S. D. Bader \\ Materials Science Division \\ Argonne National Laboratory \\ Argonne, II 60439
}

We present results on the magnetic reversal process in epitaxial Sm-Co $(1 \overline{1} 00) / \mathrm{TM}(\mathrm{TM}=$ $\mathrm{Fe}, \mathrm{Co}$ ) bilayer films prepared via magnetron sputtering onto $\mathrm{Cr}$-buffered single-crystal $\mathrm{MgO}$ substrates. The magnetically hard $\mathrm{Sm}$-Co films have 20-T uniaxial anisotropy and coercivities $>3$ $\mathrm{T}$ at room temperature. The magnetization of the soft layer is pinned at the interface to the hardmagnet layer and switches reversibly as expected for an exchange-spring magnet. With increasing soft layer thickness, the coercive field of the hard layer becomes significantly less than that of a single layer. We also present numerical solutions of a one-dimensional model that provide the spin configuration for each atomic layer. Comparison of the experimental results with the model simulations indicates that the exchange-spring behavior of our bilayer films can be understood from the intrinsic parameters of the hard and soft layers.

* Present address: IBM Almaden Research Center, 650 Harry Rd., San Jose, CA 95120-6099. 
AD-01

The exchange-spring magnets, which are composed of suitably nano-dispersed hard and soft magnetic phases, have been the subject of many recent studies. ${ }^{1-8}$ Exchange interaction between the two magnetic phases results in enhancement of the remanence and the energy-product. Although future application of exchange-spring magnets will most likely be based on nanodispersed composite geometries, ${ }^{8}$ to obtain greater insights into the coercivity mechanism and magnetization reversal process in these materials, it is advantageous to devise a simpler structure with well-defined properties to isolate the various contributions that are often masked by the structural complexities of a random two-phase system. Theoretical calculations by Skomski and Coey ${ }^{6.7}$ predicted that, in superlattice structures consisting of exchange coupled soft layers and aligned hard-magnet layers, a giant energy-product of 120 MG Oe (about three times that of commercially available permanent magnets) is attainable.

It is now possible to fabricate high-quality permanent magnet thin films with well-defined crystallographic orientation and close-to-intrinsic magnetic properties. ${ }^{9}$ For example, epitaxially grown Sm-Co films can have the c-axis of the hcp structure lying in-plane, giving rise to strong uniaxial in-plane anisotropy. ${ }^{10}$ These films, when incorporated into the bilayer structure with transition metal (TM) soft-magnet layers, provide a model system in which the magnetization rotation process of the exchange-coupled TM layers can be studied with the applied field both parallel and perpendicular to the anisotropy axis of the hard-layer. In this paper we examine the magnetization reversal processes in epitaxial hard/soft $\mathrm{Sm}-\mathrm{Co}(1 \overline{1} 00) / \mathrm{TM}$ bilayers $(\mathrm{TM}=\mathrm{Fe}$ and Co). To obtain greater insight into the switching of both the soft and hard layers, we use a simple one-dimensional atomic model to simulate the spin profile and the magnetization hysteresis loops. Comparison of the experimental results with the model simulations indicates that the exchangespring behavior can be understood from the intrinsic parameters of the hard and soft layers.

The Sm-Co $(1 \overline{1} 00) / \mathrm{TM}$ bilayers are grown via dc magnetron sputtering onto single-crystal $\mathrm{MgO}(110)$ substrates coated with an epitaxial $200-\AA \mathrm{Cr}(211)$ buffer layer. The $200-\AA \mathrm{Sm}-\mathrm{Co}$ layers are deposited by co-sputtering from separate $\mathrm{Sm}$ and $\mathrm{Co}$ sources with a nominally $\mathrm{Sm}_{2} \mathrm{Co}_{7}$ 
concentration at a substrate temperature $T_{S}=600^{\circ} \mathrm{C}$ as outlined in Refs. 9 and 10. The TM layers are then grown at $T_{S}=300-400^{\circ} \mathrm{C}$ with thicknesses of $25-200 \AA$ and capped with a $50-\AA \mathrm{Cr}$ layer. The magnetic properties were measured using a 7-Tesla SQUID magnetometer equipped with both longitudinal and transverse coils, and the longitudinal magneto-optic Kerr effect (MOKE) using ppolarized, 633-nm light.

The structural and magnetic characterization of $\operatorname{Sm}-\operatorname{Co}(1 \overline{1} 00)$ films grown onto $\operatorname{Cr}(211)$ buffer layers are described in detail in Ref. 10. The epitaxial relation for the Sm-Co(1100) films is $\mathrm{Sm}-\mathrm{Co}[0001]\|\mathrm{Cr}[01 \overline{1}]\| \mathrm{MgO}[001]$, resulting a uniaxial in-plane structure with the magnetic easy axis parallel to the Sm-Co c-axis. The films exhibit strong uniaxial in-plane anisotropy consistent with the expected $c$-axis anisotropy. For $\mathbf{H}$ parallel to the Sm-Co easy axis-(MgO[001]), a square loop is observed with a coercive field $\mathrm{H}_{\mathrm{C}}$ of $3.4 \mathrm{~T}$ for this film. The coercivity increases to $7.3 \mathrm{~T}$ at $25 \mathrm{~K}$. For $\mathbf{H}$ applied in the orthogonal in-plane direction, a sheared hard-axis loop is measured. The anisotropy field, estimated from extrapolating the hard-axis loop to saturation, is $\approx 20 \mathrm{~T}$, close to the known anisotropy value of bulk Sm-Co. These high-quality hard magnetic layers form the basis of this study.

Shown in Fig. 1 are room-temperature easy-axis hysteresis loops of the $\mathrm{Sm}-\mathrm{Co}(200 \AA) / \mathrm{Fe}$ bilayers with different $\mathrm{Fe}$ layer thickness. For a $25-\AA \mathrm{Fe}$ layer, a square easy-axis loop is measured, indicating that the Fe layer is strongly coupled to the underlying Sm-Co film and that the two layers switch as a unit. As the result of coupling between the layers, the coercivity $\mathrm{H}_{\mathrm{c}}=1.7 \mathrm{~T}$ is only $\sim 50 \%$ of that of the isolated Sm-Co film. For the 100- and $200-\AA$ Fe layers the loops change shape quite significantly. For $\mathbf{H}$ applied parallel to the easy axis, separate switching transitions for the Fe and Sm-Co layers are observed. This is similar to that observed in Refs. 11 14 but with much thinner soft layers in the present samples. The switching fields for the Sm-Co layers $(0.6-0.7 \mathrm{~T})$ are similar for the $100-$ and $200-\AA \mathrm{Fe}$ layers and are only $20 \%$ of that of the isolated $\mathrm{Sm}$-Co film value.

Shown in Fig. 2a are the hysteresis loop and recoil curves for the $100-\AA$ Fe layer film measured using the MOKE technique. As a result of the finite penetration of the light, the MOKE 
measurements are dominated by the top Fe layer. At low reverse fields, the $\mathrm{Fe}$ layer is pinned by the underlying Sm-Co layer. The Fe layer starts to switch at the exchange field $\mathrm{H}_{\mathrm{ex}}=0.22 \mathrm{~T}$. Above $\mathrm{H}_{\mathrm{ex}}$, a sharp drop in the magnetization occurs as the Fe layer starts to rotate away from alignment with the hard layer. The rotation is fully reversible since the recoil curves coincide with the demagnetization curve. At reverse fields $>0.5 \mathrm{~T}$, the recoil curves deviate from the demagnetization curve as the hard layer begins to switch irreversibly and the Fe does not recover full remanence on recoil. A quantitative assessment of the irreversibility is the irreversible magnetization change $\Delta M_{i r}=M_{r}-M_{d}(H)$ where $M_{r}$ is the remanent magnetization and $M_{d}(H)$ the field-demagnetization remanence after recoiling from reversal fieid $\mathrm{H}$. Shown in Fig. $2 \mathrm{~b}$ is $\Delta \mathrm{Mi}_{\mathrm{ir}}$ $12 \mathrm{M}_{\mathrm{r}}$ as a function of the reverse field. The magnetization is fully reversible $\left(\Delta \mathrm{M}_{\mathrm{ir}}=0\right)$ up to $0.5 \mathrm{~T}$, where the Sm-Co layer switches and $\Delta \mathrm{M}_{\mathrm{irr}}$ begins to increase sharply.

To obtain greater insight into the switching of both the soft and hard layers, we use the simple one-dimensional atomic model where the bilayer is treated as a chain of spins normal to the layers, each spin is the sum of total moments in an atomic layer ${ }^{11,13,15}$. The total energy of the system is given by:

$$
E=-\sum_{i=1}^{N-1} \frac{A_{i, i+1}}{d^{2}} \cos \left(\theta_{i}-\theta_{i+1}\right)-\sum_{i=1}^{N} K_{i} \cos ^{2}\left(\theta_{i}\right)-\sum_{i=1}^{N} H M_{i} \cos \left(\theta_{i}-\theta_{H}\right),
$$

where the rotation angle for the $\mathrm{i}$-th layer $\theta_{\mathrm{i}}$ is measured relative to the easy-axis direction of the hard layer, $\theta_{\mathrm{H}}$ is the angle between the field and the easy axis, $A_{i}, K_{i}, M_{i}$, $d$ are the exchange constants, uniaxial anisotropy constants, magnetic moments and inter-plane distance $(=2 \AA)$, respectively. The equilibrium spin configuration for a given field is determined by minimizing Eq. 1. To calculate this configuration we employ an iterative approach outlined by Camley. ${ }^{16}$ Details of the modeling will be published elsewhere. ${ }^{17}$

Shown in Fig. 3a is the comparison of the calculated $\mathrm{Sm}-\mathrm{Co} / \mathrm{Fe}(200 \AA)$ demagnetization curves to the ones measured at $25 \mathrm{~K}$. Included are both the longitudinal and transverse 
magnetization with respect to the applied field. The parameters for the hard layer are $A_{h}=1.2 \times 10^{-6}$ ergs $/ \mathrm{cm}, \mathrm{K}_{\mathrm{h}}=5 \times 10^{7} \mathrm{ergs} / \mathrm{cm}^{3}, \mathrm{M}_{\mathrm{h}}=550 \mathrm{emu} / \mathrm{cm}^{3} ;$ for the soft layer, $\mathrm{A}_{\mathrm{s}}=2.8 \times 10^{-6} \mathrm{ergs} / \mathrm{cm}, \mathrm{K}_{\mathrm{s}}=10^{3}$ $\mathrm{ergs} / \mathrm{cm}^{3}, M_{s}=1700 \mathrm{emu} / \mathrm{cm}^{3}$, the interface exchange constant $A_{i n t}=1.8 \times 10^{-6} \mathrm{ergs} / \mathrm{cm}$ and $\theta_{H}=3^{\circ}$. The values of $K_{h}$ and $M_{h}$ were estimated from magnetization measurements on the Sm-Co films. The value of $A_{i n t}$ was set intermediate to exchange coupling of the hard and soft layers. The calculation reproduces the $\mathrm{H}_{\mathrm{ex}}$ value, the field dependence of both the longitudinal and transverse magnetization, as well as the switching field of the Sm-Co layer at $\approx 1.5 \mathrm{~T}$. The irreversible switching of the hard layer is expected to be the least reliable parameter determined by this type of modeling and the close agreement in Fig. 3a is rather fortuitous. Shown in Fig. $3 b$ is the spin configuration at various fields for the calculated magnetization in Fig. 3a. The distribution of moments is consistent with the expectation that the Fe located away from the interface rotates more. For the configuration at $\mathrm{H}=0.14 \mathrm{~T}$ the average $\mathrm{Fe}$ angle is $\approx 90^{\circ}$ resulting in the maximum in the transverse moment with applied field. As the field increases, the turn angle of the Fe spins away from the interface increases and the surface layers align with the field. At $\mathrm{H}=1.45 \mathrm{~T}$ (just below the switching of the hard layer), the top $\approx 120 \AA$ of the Fe layer aligns with the field.

The calculations indicate that the hard layer is also significantly perturbed by the rotation of the Fe layers, in agreement with Mibu et al. ${ }^{11}$ As $\mathrm{H}$ increases, the interfacial Sm-Co spin is also increasingly rotated and a domain wall is slowly introduced into the hard layer. This domain wall, nucleated by the Fe layer, reverses the Sm-Co layer at a field well below that expected for an isolated Sm-Co film. The reduction of the switching field of the hard layer was explained in Ref. 1. As the field increases, the domain wall in the soft layer is compressed and its energy increases. The wall energy in the soft layer $\gamma_{s}$, assuming no anisotropy, is expected to vary as $\sqrt{\mathrm{A}_{s} \mathrm{M}_{\mathrm{s}} \mathrm{H}}$. The energy of a domain wall in the hard layer is $\gamma_{h}=4 \sqrt{A_{h} K_{h}}$. As the field increases such that $\gamma_{s}$ becomes greater than $\gamma_{h}$, the domain wall in the soft layer moves into, and switches, the hard layer. 
The field at which the hard layer switches should roughly scale as $A_{h} K_{h} / A_{s} M_{s}$ and is significantly lower than that for the isolated film.

The switching of the hard layer also depends on the thickness of the soft layer. With the parameters obtained from the fit, we can simulate a series of hysteresis loops for different $\mathrm{Fe}$ layer thicknesses. This is shown in Fig. 4 where the calculated Sm-Co switching fields are compared to those of the $\mathrm{Sm}-\mathrm{Co} / \mathrm{Fe}$ samples measured at $25 \mathrm{~K}$. The switching for the hard layer initially decreases dramatically with increasing Fe thickness. Even a 10- $\mathrm{A}$ Fe layer reduces the calculated coercivity of the hard layer by a factor of 3 . When the Fe-layer thickness become comparable to the width of a domain wall, the switching of the hard layer becomes independent of the Fe layer thickness. The general trend in the data is well reproduced by the simulations.

The temperature dependence of the $\operatorname{Sm}-\operatorname{Co}(200 \AA) / \operatorname{Co}(100 \AA)$ bilayer is shown in Fig. 5 . At low temperatures, separate switching transitions are observed for the Sm-Co and Co layers at $\mathrm{H}_{\mathrm{irr}}$ and $\mathrm{H}_{\mathrm{ex}}$, respectively. The exchange field for the bilayer structure with Co layers is significantly higher than that with Fe layers of comparable thickness. This behavior arises from the intrinsic magnetic anisotropy of the Co layer. The $c$-axis Co anisotropy stabilizes the Co layer either parallel or antiparallel to the Sm-Co film and results in an enhanced Co switching field. The Co layer reverses by nucleating a $180^{\circ}$-domain wall. As long as the Sm-Co does not switch, the $\mathrm{H}_{\mathrm{ex}}$ is given by the requirement that the energy $\operatorname{cost}(\gamma)$ in creating a domain wall be balanced by the gain in Zeeman energy $\left(H M_{s} t\right)$, i.e., $H_{e x}=\gamma / M_{s} t$. where $t$ is the Co layer thickness. A rough estimate gives $\mathrm{H}_{\mathrm{ex}} \approx 0.7 \mathrm{~T}$, in reasonable agreement with the experiment. The exchange field $\mathrm{H}_{\mathrm{ex}}$ remains rather temperature-insensitive whereas the $\mathrm{H}_{\text {irr }}$ decreases with increasing temperature, suggesting that the hard layer switches via some thermally assisted reverse domain nucleation and wall de-pinning. At temperatures above $250 \mathrm{~K}$, square easy-axis loops are observed as the Sm-Co and Co layers switch simultaneously a nucleation field $\mathrm{H}_{\mathrm{N}}$.

In conclusion, we have presented the experimental results on strongly exchange-coupled Sm-Co(1100)/TM bilayer films. The magnetically hard Sm-Co layers have an in-plane uniaxial 
anisotropy field as large as $20 \mathrm{~T}$. Such a large anisotropy field enables us to explore exchangespring behavior in a new regime where the layer thicknesses are less than $20 \mathrm{~nm}$. With increasing soft layer thickness, the coercive field of the hard layer becomes significantly less than that of a single layer. The present results can be understood from the intrinsic parameters of the hard and soft layers. A strong interlayer coupling is required to model the experimental data. With a large interfacial exchange energy (much greater than the domain wall energy), the moments in the soft layer near the interface are pinned by the hard layer, the switching of the soft layer is determined by its intrinsic properties. If the interlayer coupling was weak, the magnetization reversal of the hard layer would not be affected by the presence of the soft layer.

This work was supported by the U.S. Department of Energy, Basic Energy SciencesMaterials Science, under Contract No. W-31-109-ENG-38. 


\section{References}

${ }^{1}$ E. F. Kneller and R. Hawig, IEEE Trans. Mag. 27, 3588 (1991).

2 J. Ding, P. G. McCormick, and R. Street, J. Magn. Magn. Mater. 124, L1 (1993).

${ }^{3}$ L. Withanawasam, G. C. Hadjipanayis, and R. F. Krause, J. Appl. Phys. 75, 6646 (1994).

${ }^{4}$ I. A. Al-Omari and D. J. Sellmyer, Phys. Rev. B 52, 3441 (1995).

${ }^{5}$ T. Schrefl, H. F. Schmidts, J. Fidler, and H. Kronmuller, IEEE Trans. Magn. 29, 2878 (1993).

${ }^{6}$ R. Skomski and J. M. D. Coey, Phys. Rev. B48, 15812 (1993).

${ }^{7}$ R. Skomski, J. Appl. Phys. 76, 7059 (1994).

${ }^{8}$ J. M. D. Coey, Solid State Communications 102, 101 (1997).

${ }^{9}$ E. E. Fullerton, C. H. Sowers, J. P. Pearson, S. D. Bader, X. Z. Wu and D. Lederman, Appl. Phys. Lett. 69, 2438 (1996).

${ }^{10}$ E. E. Fullerton, J. S. Jiang, C. Rehm, C. H. Sowers, S. D. Bader, J. B. Patel and X. Z. Wu, Appl. Phys. Lett. in press (1997).

${ }^{11}$ K. Mibu, T. Nagahama and T. Shinjo, J. Magn. Magn. Mater. 163, 75 (1996).

${ }^{12}$ D. Givord, J. Betz, K. Mackay, J. C. Toussaint, J. Voiron and S. Wüchner, J. Magn. Magn. Mater. 159, 71 (1996).

${ }^{13}$ S. Wüchner, J. C. Toussaint and J. Voiron, Phys. Rev. B 55, 11576 (1997).

${ }^{14}$ Y. Suzuki, R. B. van Dover, E. M. Gyorgy, J. M. Phillips and R. J. Felder, Phys. Rev. B 53, 14016 (1996).

${ }^{15}$ E. Goto, N. Hayashi, T. Miyashita and K. Nakagawa, J. Appl. Phys. 36, 2951 (1965).

${ }^{16}$ R. E. Camley, Phys. Rev. B 35, 3608 (1987).

${ }^{17}$ Eric E. Fullerton, J. S. Jiang, M. Grimsditch, C. H. Sowers, and S. D. Bader, Phys. Rev. B, submitted. 


\section{Figure Captions}

Figure 1: Room-temperature magnetic hysteresis loops for single layer $\mathrm{Sm}-\mathrm{Co}$ and $\mathrm{Sm}-\mathrm{Co} / \mathrm{Fe}$ bilayer films with different $\mathrm{Fe}$ thickness with $\mathrm{H}$ parallel to the easy-axis directions.

Figure 2: Room-temperature magnetic properties of $\mathrm{Sm}-\mathrm{Co} / \mathrm{Fe}$ bilayer films measured by MOKE. (a) Hysteresis loop (solid curve) and recoil curves (open circle) measured with $\mathrm{H}$ parallel to the easy-axis directions for the $100-\AA \mathrm{Fe}$ film. (c) The irreversible magnetization $\Delta \mathrm{M}_{\mathrm{ir}} v s$. reverse field measured with $\mathrm{H}$ parallel to the easy axis.

Figure 3: (a) Low-temperature $(25 \mathrm{~K})$ demagnetization curves for the $\mathrm{Sm}-\mathrm{Co} / \mathrm{Fe}(200 \AA)$ film compared to the model calculation (solid line) described in the text. The longitudinal and transverse components of the magnetization are given by the circles and triangles, respectively. (b) Representative spin configuration determined from the model calculation shown in (a). Open circles are Fe spins and filled circles are $\mathrm{Sm}$-Co spins. The free Fe surface is located at zero and the $\mathrm{Sm}-\mathrm{Co} / \mathrm{Fe}$ interface is at $200 \AA$.

Figure 4: Calculated irreversibility field for $\mathrm{Sm}-\mathrm{Co} / \mathrm{Fe}$ films as a function of Fe thickness compared to the measured values for the Sm-Co/Fe bilayer films measured at $25 \mathrm{~K}$.

Figure 5: (a) Easy-axis hysteresis loops for the $\operatorname{Sm}-\operatorname{Co}(200 \AA) / \operatorname{Co}(100 \AA)$ measured at different temperatures. (b) The switching fields plotted as a function of temperature. 


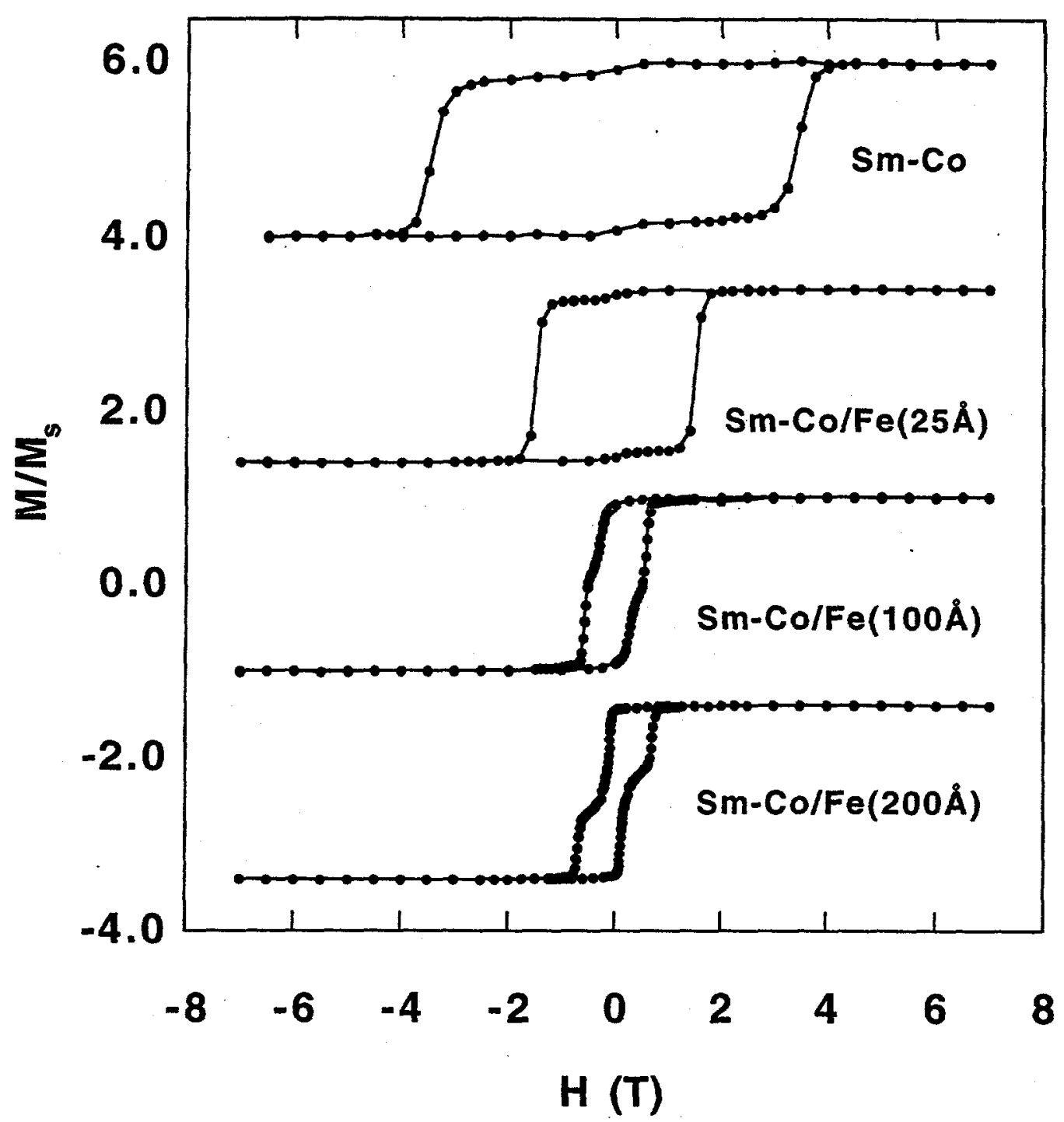

Figure 1 


\section{AD-01}
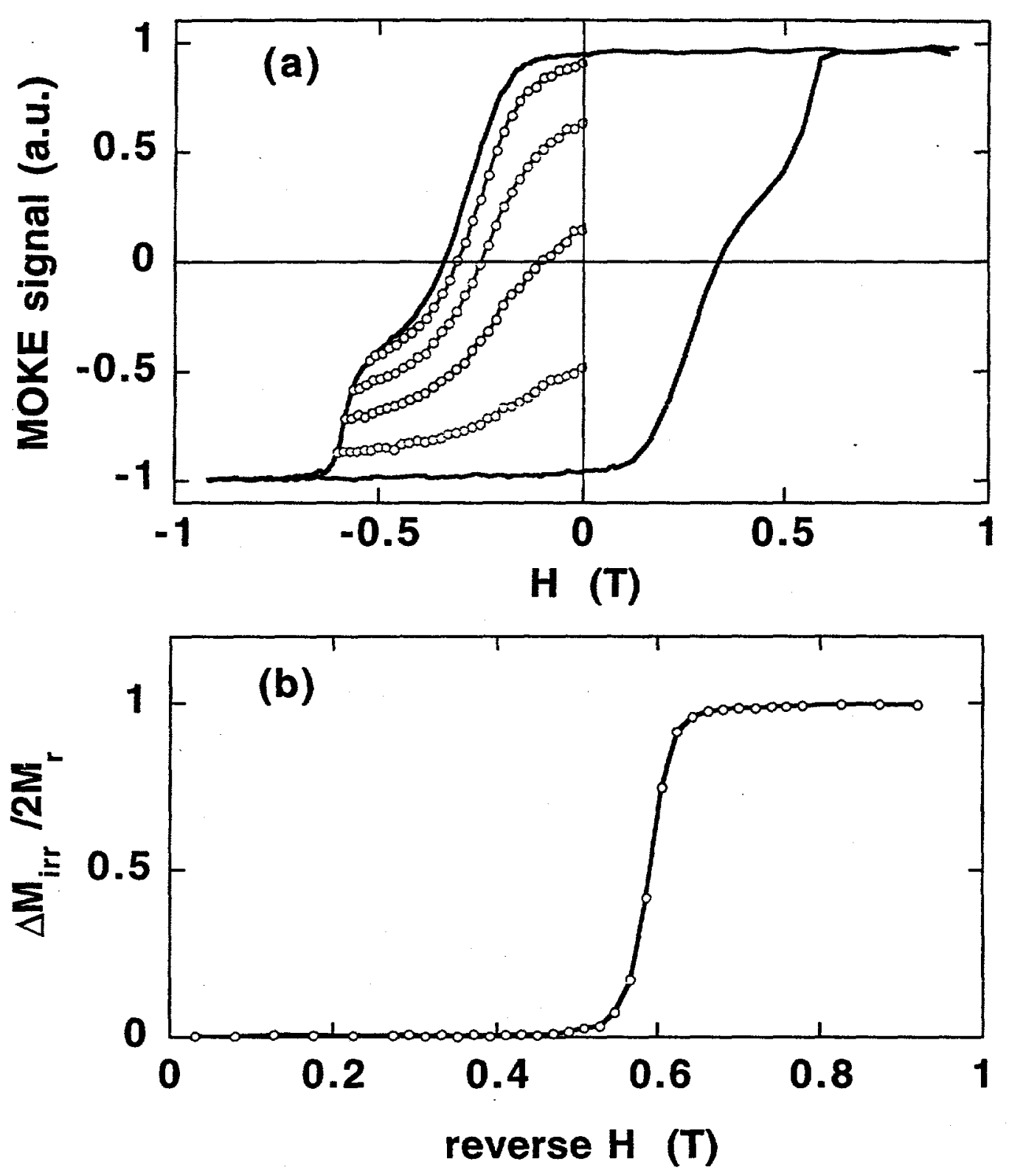

Figure 2 

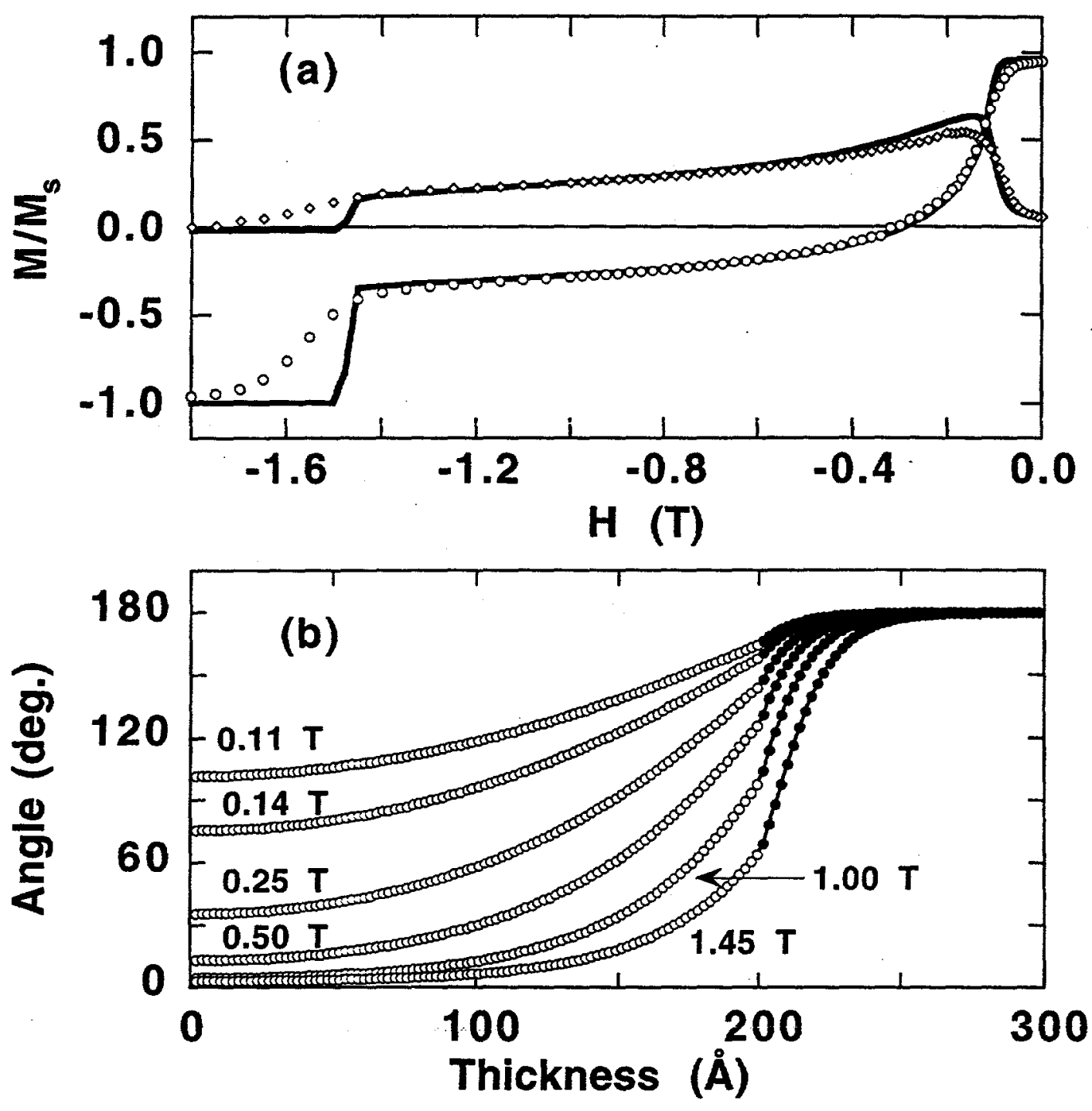

Figure 3 


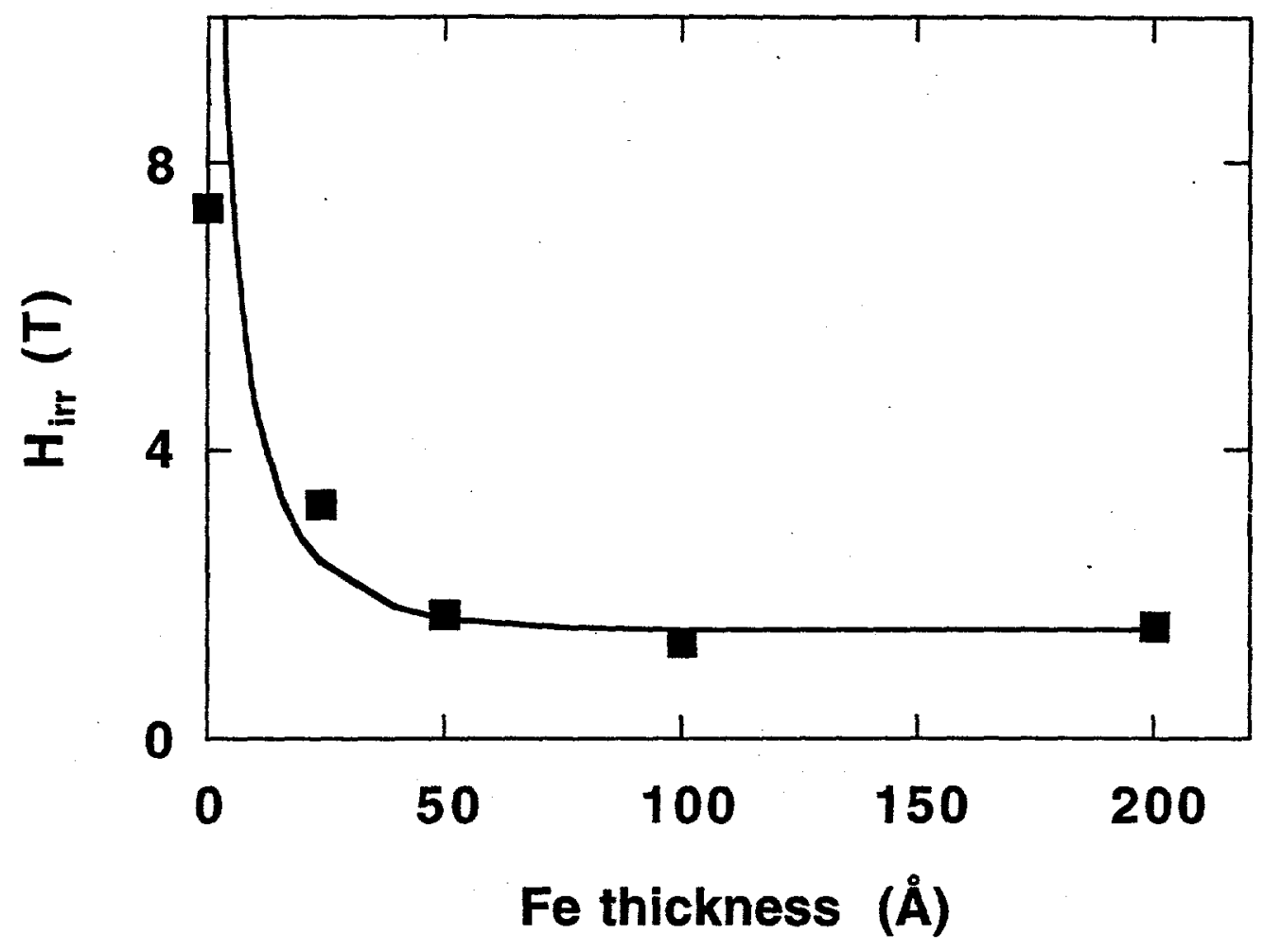

Figure 4 

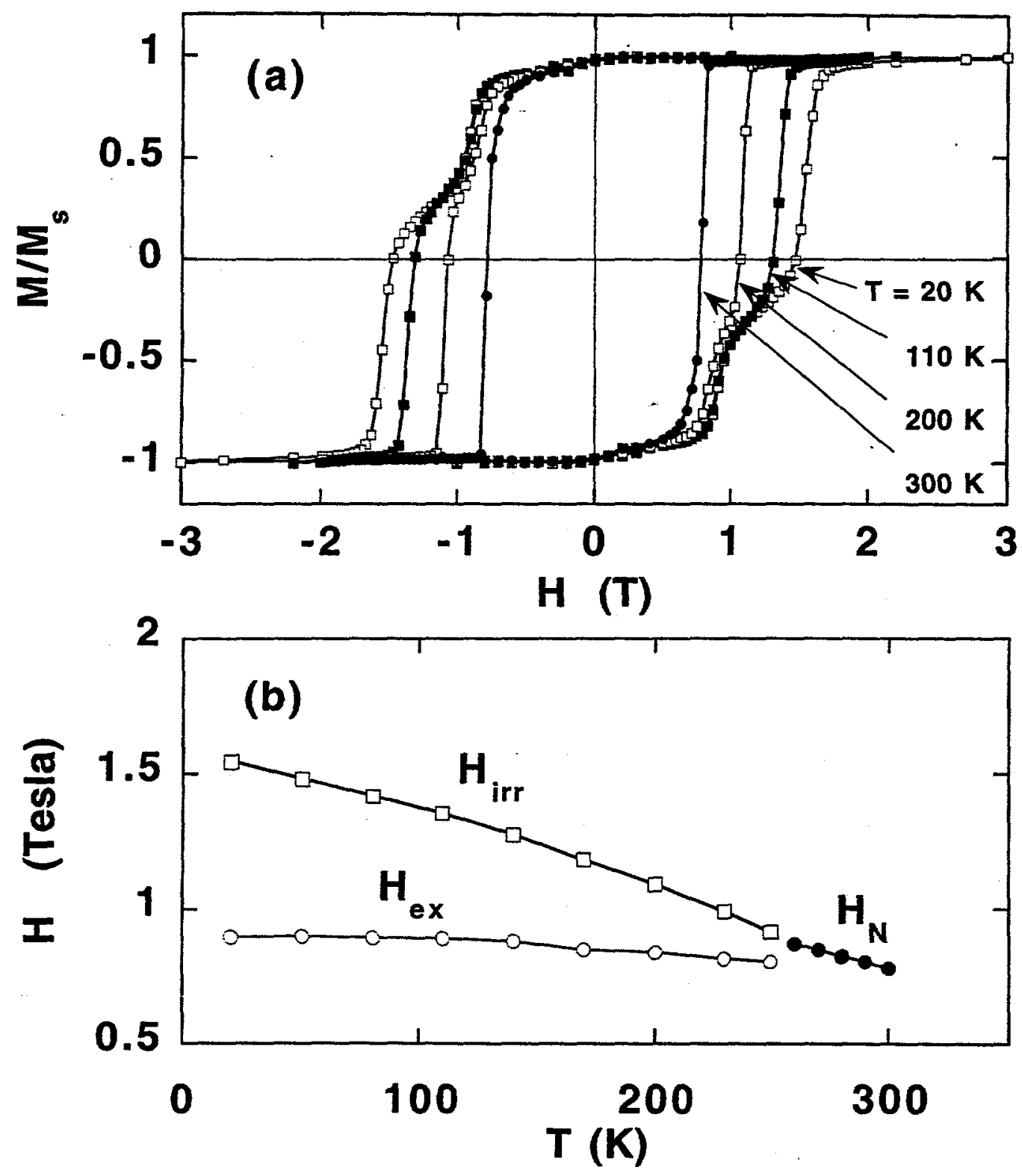

Figure 5 